\title{
FIELD TRIALS OF A SELF-AdJUSTING SEED FINGER SYSTEM TO IMPROVE GIN TURN-OUT AND FIBER PROPERTIES
}

\author{
Andrzej Krajewski ${ }^{1}$, Stuart Gordon ${ }^{2, *}$ David Fox ${ }^{3}$ \\ ${ }^{1}$ RMIT University, Melbourne, Victoria, Australia. \\ ${ }^{2}$ CSIRO Agriculture and Food, Waurn Ponds, Victoria, Australia. \\ ${ }^{3}$ CSIRO Manufacturing, Waurn Ponds, Victoria, Australia. \\ *Correspondence: stuart.gordon@csiro.au.
}

\section{HighLIGHTS}

- A dynamic seed finger system that automatically responds to pressure along the seed roll was demonstrated.

- Seed roll density is dynamic and affected by the seed finger partition angle.

- At low and medium pressures, the system improved energy consumption, gin turn-out, and fiber quality.

ABSTRACT. Previously, we reported a prototype system whereby the position of the seed fingers in a saw gin could be adjusted continuously to affect the amount of residual lint on the ginned seed and provide more fiber (increased gin turnout). The system combines partitioned seed fingers pivoted on a shaft that are controlled by electric actuators able to adjust the angular position of each seed finger partition according to the load exerted by the seed roll. That previous work noted that as the seed finger angle and load on the seed roll were increased, more residual lint was removed. Initial tests showed differences of up to $1.4 \%$ less residual lint with no impact on seed damage after the seed finger angle of the partitions was increased (in unison) to the maximum value. Following those findings, a dynamic feedback mechanism for automatically adjusting the seed finger partitions according to a relationship between the load applied by the seed roll and the seed finger angle was developed. In this article, we describe preliminary tests of the automatic mode (auto mode), i.e., the application of auto-modulating low $\left(6^{\circ}\right)$, medium $\left(12^{\circ}\right)$, and high $\left(18^{\circ}\right)$ loads to the seed roll in a commercial saw gin during seasonal production. The resulting effects on residual lint (turn-out), seed damage, and fiber quality in each mode were measured. The best results in terms of energy savings, reduced residual fiber on the ginned seed, seed damage, fiber length, and color grade were found when low $\left(6^{\circ}\right)$ to medium $\left(12^{\circ}\right)$ loads were applied across the seed roll.

Keywords. Automation, Cotton, Ginning, Load sensor, Roll box, Seed finger, Seed roll.

S

eed fingers are an important part of a gin stand and are used to control the density of the accumulated seed roll and the rate of seed drop during ginning. They are typically a pivoted array of evenly distributed prongs or a flap (or lambrequin) extending toward the gin saws from the top of the huller ribs that acts to support the seed roll, which is the revolving mass of seed cotton that accumulates on top of the gin saws and seed fingers. The seed fingers support the seed roll and present seed cotton that has not been fully ginned back onto the saws for ginning. From an operational point of view, the seed fingers are undeveloped in their ability to be dynamically controlled for the benefit of the ginner, i.e., improved quality and increased gin turn-out. Currently, the seed finger position is set

\footnotetext{
(c) (1) $\Theta$ The authors have paid for open access for this article. This C. ${ }_{\mathrm{BY}} \mathrm{NC}_{\mathrm{ND}}$ work is licensed under a Creative Commons AttributionNonCommercial-NoDerivatives 4.0 International License https://creative commons.org/licenses/by-nc-nd/4.0/

Submitted for review on 6 February 2021 as manuscript number MS 14489; approved for publication as a Research Article by the Machinery Systems Community of ASABE on 2 June 2021.
}

manually by the ginner, who fixes the position to one of three or four available settings. The decision to adjust the seed finger position is based on the seed roll being able to turn unimpeded without excessive drop of partially ginned seed and based on the gin operator's experience in noting these effects. If the setting is suitable, it is often left in that position for an entire ginning season. The setting is not routinely adjusted because of the inconvenience of stopping the gin stand to make the adjustment. Moreover, there are no quantifiable feedback mechanisms, other than visual inspection of the seed flow beneath the gin and the gin motor current, to determine the appropriateness of the load or pressure that should be applied to the seed roll.

In earlier work, Krajewski et al. (2016) reported the load ( $\mathrm{kg}$ force) applied by the seed roll across the three seed finger partitions was unequal and dynamic, and that greater load was applied to the center partition, with lower forces on the right and left partitions. This agreed with earlier observations by Bagshaw (2011), who found seed roll density was greater across the middle partition and that there was much less seed drop in the middle than in the right and left sides of 
the gin stand. We also noted that as the angle of the partition was increased to its maximum pivot of $30^{\circ}$, i.e., the seed roll density was increased, more lint was removed from the seed. Up to $1.4 \%$ less residual lint on the ginned seed was recorded for seed cotton ginned with the seed finger angle at its maximum $\left(30^{\circ}\right)$, without any impact on seed damage. As well as reducing residual lint, increasing the angle of the seed fingers (in unison) increased the load applied to the seed roll and surprisingly reduced the gin motor power consumption.

After this initial work, a feedback mechanism to automatically adjust the seed finger mechanism according to a set applied load was developed and tested. As described in our previous work, a set of motor controllers (Festo MMO-STC5-1-DIOP) were used to control stepper motors that regulate the angular position of the three seed finger partitions via electrical actuators. Load cells (Burster 8415) connected to each partition provide feedback on the load exerted on the seed fingers by the weight of the seed roll. A fan-less lowpower PC was used to acquire and process the load cell measurements to actuate the required partition angle. A block diagram of the automated seed finger system is shown in figure 1 .

Load measurements were linked via the following sequence of equations in the system software:

$$
\text { Load partition }(\alpha)=A \times \text { Elevation }(\alpha)+B
$$

Res Lint partition $(\alpha)=C \times$ Elevation $(\alpha)+D$

$$
\text { Elevation }(\alpha)=\frac{(\text { Load partition }(\alpha)-B)}{A}
$$

$$
\text { Elevation }(\alpha)=\frac{(\text { Res Lint partition }(\alpha)-D)}{C}
$$

Hence:

$$
\frac{(\text { Res Lint partition }(\alpha)-D)}{C}=\frac{(\text { Load partition }(\alpha)-B)}{A}
$$

Therefore:

Res Lint partition $(\alpha)=($ Load partition $(\alpha)-B) \times C$

where $\alpha$ is the seed finger angle calculated from the seed finger partition's lowest position, and $A, B, C$, and $D$ are empirically calculated coefficients for each partition (left, center, and right). The coefficient values represent the incumbent friction within the system at set throughputs and the initial forces on the load cells exerted by the mechanical arrangement.

As described, the system software was programmed to automatically control the load applied to each seed finger partition within defined ranges. The ranges were determined by one or all or the following factors: the observed residual lint on the ginned seed, the seed finger pivot angle, and/or the gin motor current (power) draw. The system software was written in LabVIEW and run on a modified Microsoft Windows 7 platform. The automatic mode (auto mode) system, in which stepper motors automatically adjust the angle of each seed finger partition according to feedback from the load cells and/or motor current draw, is illustrated in figure 2 . The auto mode system provides a timed period to determine the load on each partition and the required angle to

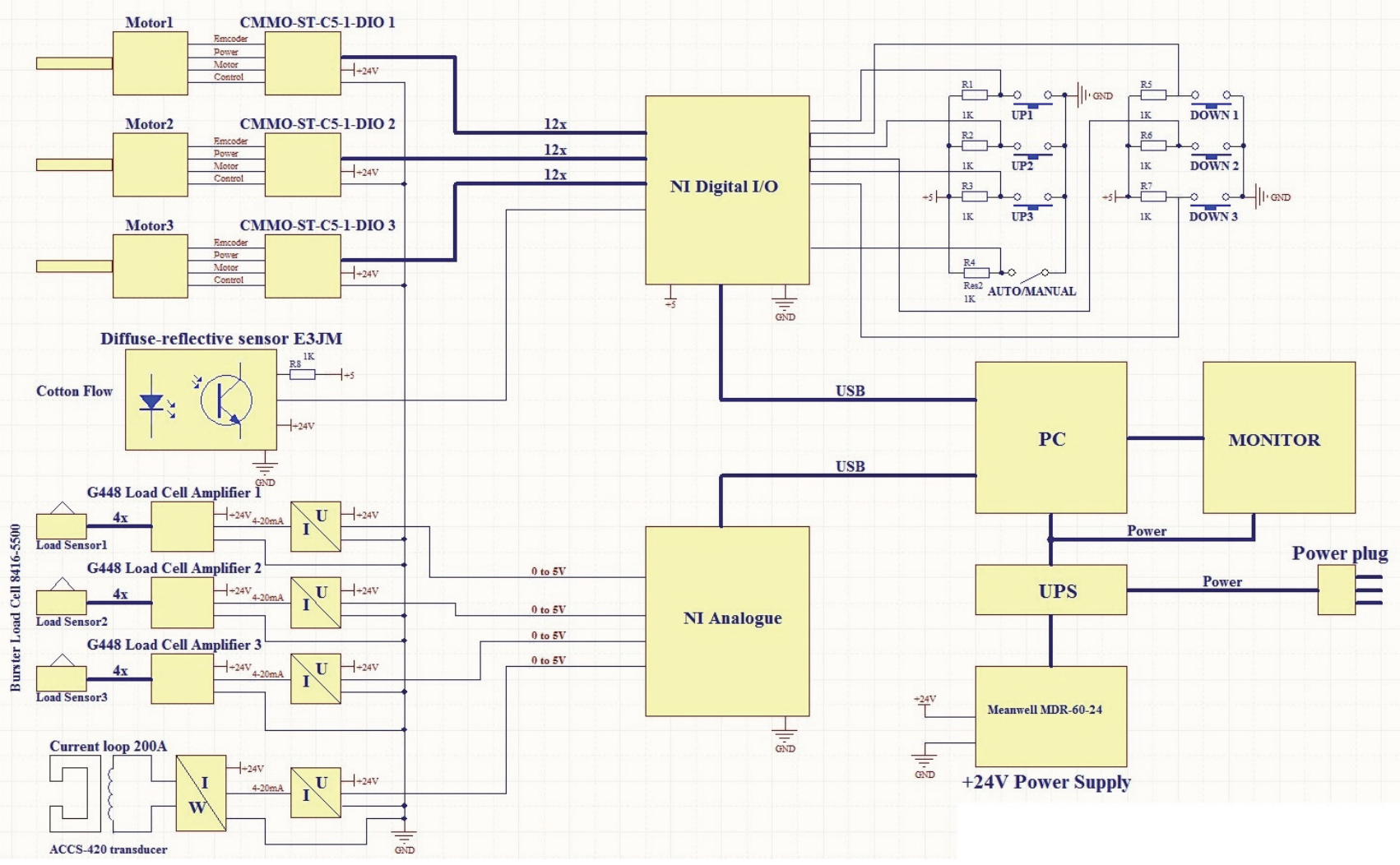

Figure 1. Block diagram of automated seed finger system. 


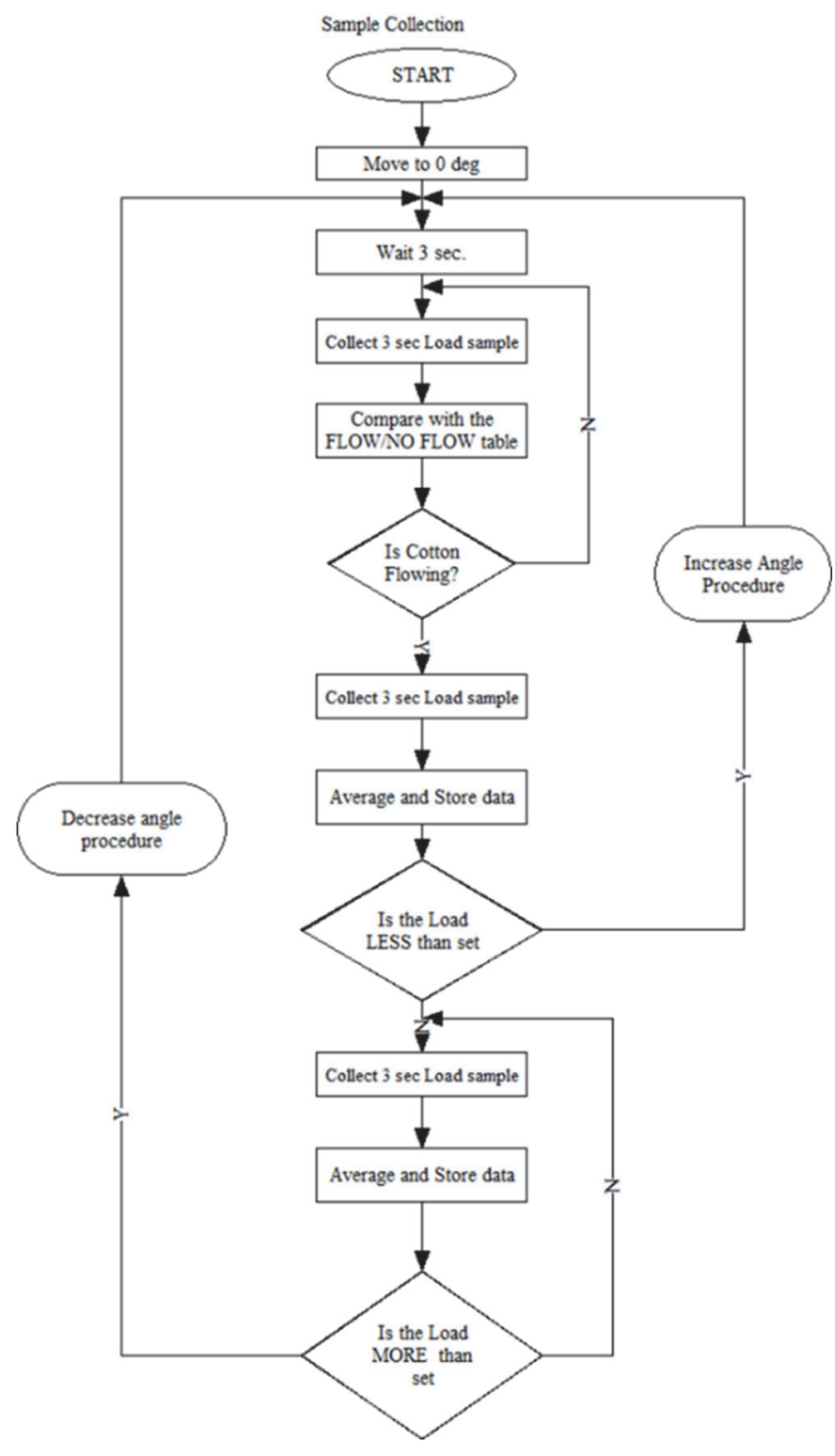

Figure 2. Flowchart of auto mode for a single partition.

achieve that load. The load and/or current draw is then averaged over this period (a period of $3 \mathrm{~s}$ is indicated in fig. 2) and fed into a feedback loop to enable the system to decide to change the seed finger elevation angle. This article reports preliminary test data for the auto mode system and the consequences of a partitioned and automatically modulated load across the seed roll at three nominal settings (low, medium, and high) on current draw, fiber properties, and gin turn-out as measured by residual lint determination.

\section{MATERIALS AND MeTHODS}

Tests of the automated system were carried out in a Golden Eagle Series 161 gin located in Warren, New South Wales, Australia $\left(31.7^{\circ} \mathrm{S}, 147.8^{\circ} \mathrm{E}\right)$. The gin has five stands and a capacity of 90 bales per hour (18 bales per stand), usually with clean irrigated, picker-harvested upland cotton. The seed finger system was designed and built by CSIRO and fitted into the fifth (end) stand of the gin, as reported previously by Krajewski et al. (2016). Each stand, including the modified stand, uses a seed conveyor tube system common to Continental Eagle Series 161 gins to drive the seed roll and provide additional discharge capacity for ginned seed. The ginning rates were not purposely adjusted during the trials and were fixed at 15 bales per stand per hour. The seed cotton used in the experiments was an Australian upland variety (Sicot 74BRF) from a commercial irrigated, well defoliated, spindle-harvested crop sown in late 2014 in the same area as the gin, harvested in late March 2015, and ginned for these trials in mid-April 2015.

The load values for the auto mode system were set using load values reported by Krajewski et al. (2016), i.e., the load ( $\mathrm{kg}$ force) values recorded across each partition as the partitions were moved from $0^{\circ}$ (minimum) to $30^{\circ}$ (maximum). Table 1 lists the nominal load values for each mode (low, medium, and high). Each mode is defined according to the load rather than the partition angle. The partitions across the seed roll are therefore allowed to fully open and close in response to the load range associated with each mode. The force exerted on each partition's load sensor is translated into changes in voltage, with the load sensor (fig. 1) in a resistive Wheatstone bridge configuration.

Signals from the load sensors are fed into an analog-todigital converter and sent via a National Instruments (NI) analog I/O card to the system computer, where the data are saved as an Excel (.csv) file. The acquired data are then processed by the system software (written in LabView) and compared with the prescribed load for the nominal mode (low, medium, or high) by the system computer. A small microprocessor, e.g., an open-source (Arduino) logic board, would be more economical, particularly for production applications. However, using an embedded PC with an NI multifunction I/O device and the LabView programming language provided the ability for the software to be changed rapidly for testing and research. Based on the processed data, a decision is triggered to lower or raise the partition. Each partition's angle of elevation is controlled independently by the system computer, with a feedback mechanism embedded in the response algorithm using the measured load cell signal to adjust the seed finger elevation, so that the forces are as equal as possible across the partitions.

The auto mode system was logged for up to $15 \mathrm{~min}$ in each mode after an initial 10 min gin run setting period. The modes were logged consecutively from low to high over two days using the same cotton, providing two sets (trial days) of data from each mode for analysis. The weather conditions were cool and dry throughout, i.e., gin flue temperatures were similar on both days of ginning. The partition angle movement, the load cell values for each partition, and the gin motor current draw were logged during each run. The motor current values during each run were recorded with a $200 \mathrm{~A}$ generic current loop probe (ACCS-420 transducer). The

Table 1. Auto mode levels, angles, and associated load ranges for each partition of the seed finger assembly.

\begin{tabular}{cccc}
\hline Mode & $\begin{array}{c}\text { Nominal } \\
\text { Corresponding Angle } \\
\text { (degrees) }\end{array}$ & $\begin{array}{c}\text { Lower } \\
\text { Load Limit } \\
(\mathrm{kg})\end{array}$ & $\begin{array}{c}\text { Upper } \\
\text { Load Limit } \\
(\mathrm{kg})\end{array}$ \\
\hline Low & 4 to 8 & 1.9 & 2.1 \\
Medium & 10 to 14 & 2.1 & 2.3 \\
High & 16 to 20 & 2.4 & 2.5 \\
\hline
\end{tabular}


current loop probe was clamped on one of the power cables of the three-phase gin motor, with the output connected to a current-to-voltage transducer. The voltage was translated into a digital signal and sent to the system computer through via the NI analog I/O card. Motor current values were collected simultaneously with load cell values and saved as Excel (.csv) files.

In each mode, ginned seed samples were collected at intervals of 4 to $5 \mathrm{~min}$ under each partition (left, center, and right) so that three replicate samples for residual lint and seed damage determination were collected during each trial and mode. For these tests, $60 \mathrm{~g}$ subsamples of the seed from each sample were taken and any broken seeds and/or plant trash removed manually. Fifty grams $\pm 0.05 \mathrm{~g}$ of the cleaned seed was then oven-dried overnight at $105^{\circ} \mathrm{C}$. The dried subsample was cooled in a desiccator, reweighed to \pm 0.05 $\mathrm{g}$, and then washed in a glass beaker using 150 to $200 \mathrm{~mL}$ of concentrated $(98 \%) \mathrm{H}_{2} \mathrm{SO}_{4}$. The washed and denuded subsamples were drained, oven-dried for a further $4 \mathrm{~h}$ at $105^{\circ} \mathrm{C}$, and then reweighed. The difference in oven-dried weight before and after the acid bath is expressed as the residual lint removed (hydrolyzed) by the acid. Damaged seeds were manually separated from the acid-delinted seeds and weighed. Damaged seed values are expressed as the percentage of damaged seed to the total delinted and dried seed.

Fiber samples $(100 \mathrm{~g})$ were collected at the same time as the seed samples via the two inspection hatches (left and right sides) at the bottom of the Super-J duct at the back of the modified gin stand before lint cleaning. Fiber samples were classed using a high-volume instrument (HVI) line at Auscott Classing Offices in Artarmon, New South Wales, Australia, according to Australian cotton best management practice guidelines.

\section{Data Analysis}

Seed and fiber test values were subjected to a nested analysis of variance (ANOVA) using Minitab 17 to examine the effects of trial (day), partition position (left, center, or right), and auto mode (low, medium, or high) on residual lint, seed damage, and HVI fiber properties. All factors in a fully nested ANOVA are assumed to be random, so the analysis tests the hypothesis that the variance affected by each factor is zero. Three test sample replicates represented each combination of factors, i.e., trial $\times$ partition $\times$ mode. The assumed hypothesis for this analysis was that trial day will have zero variance, and the variance associated with the partition position and auto mode factors should be significant for some seed and fiber properties, nominally the ginned seed's residual lint and fiber length and trash values. Main effects plots are used to illustrate the effects of each factor. The mean partition angle, load, and motor current values per mode over the two trial days were also calculated along with the standard deviations of these means to allow general conclusions about the effects of these factors during the trials.

\section{DisCUSSION OF RESULTS \\ Seed Finger Partition Movement}

Figure 3 shows the movement responses of the three seed finger partitions during ginning using the three auto modes for the set periods over the two trial days. Figure 4 shows the corresponding load values on the partitions. Each partition started at the same nominal angle in each auto mode (table 1). Feedback from the load cells then changed the angular position of each partition, keeping the partitions within the predefined load values. Evident in figures 3 and 4 is the dynamic nature of the seed roll density as the seed roll accumulated and discharged within the roll box. Table 2 lists the mean partition angles, loads, and gin motor current recorded in each mode across the two trial days. The mean loads across the partitions matched their auto mode settings, with more variation in angular movement and load measurements at higher loads.

Overall, the loads on the partitions across the gin stand were uneven and dynamic, with a bias in load and partition angle evident on the left side. The left partition in all modes typically stayed at its minimum set angle (i.e., $8^{\circ}, 12^{\circ}$, and $18^{\circ}$ ), while the center and right partitions were consistently raised above the minimum to maintain the nominal load on the partition. This chasing of load by the center and right partitions was considered partly an effect of the modified gin stand's position as the end stand (gin stand 5), where seed cotton fed via the conveyor distributor into the extractor above the gin could be variable, depending on the production conveyed to the first four stands. This effect was evident in all modes, where gaps or stops in feed led to sharp increases in the partition angle, e.g., day 1 between 12 and $13 \mathrm{~min}$ in figure $3 \mathrm{a}$. This effect was combined with uneven seed cotton feed into the modified gin stand, which was biased to the left side of the gin apron. The left-side bias was evident in all modes, wherein partition movement occurred first on the left side before the load was transferred, or equilibrated, across the other partitions.

This input effect, which is likely to be present to some extent across all gin stands, creates an initial, uneven seed roll in terms of density that then normalizes across the roll box as seed cotton moves from high-density to low-density zones and ginned seed is discharged. The evidence of leftside bias seen in this study is that the load applied to the center partition was always below the prescribed average load, and the center partition was extended to higher angles. The right partition, while raised to higher angles, carried loads similar to the left partition. These effects were smaller for the low and medium modes but still prominent. Interestingly, the observed seed discharge was constant across the bottom of the modified gin stand in all modes.

The nominally lower density of the seed roll across the center partition in auto mode, identified by the lower applied load and increased partition angle, and the constant discharge of ginned seed across the gin stand differed from the observations reported by Bagshaw (2011) for static partitions held at a common angle. In that situation, a bridging effect across the middle partition was observed whereby the seed roll was compacted across the middle partition. 

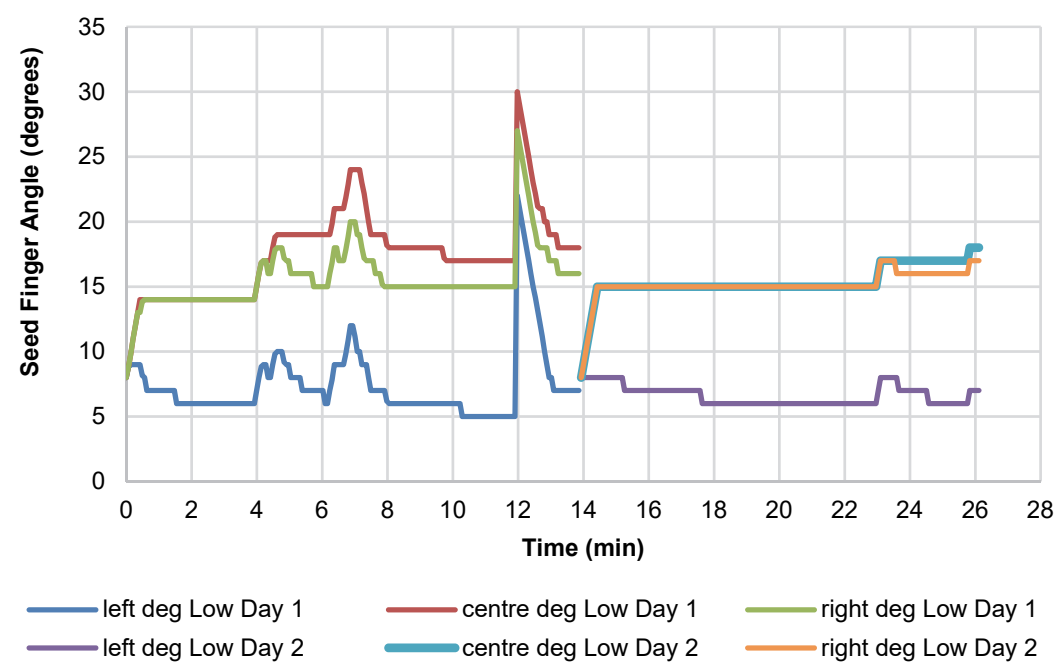

(a)
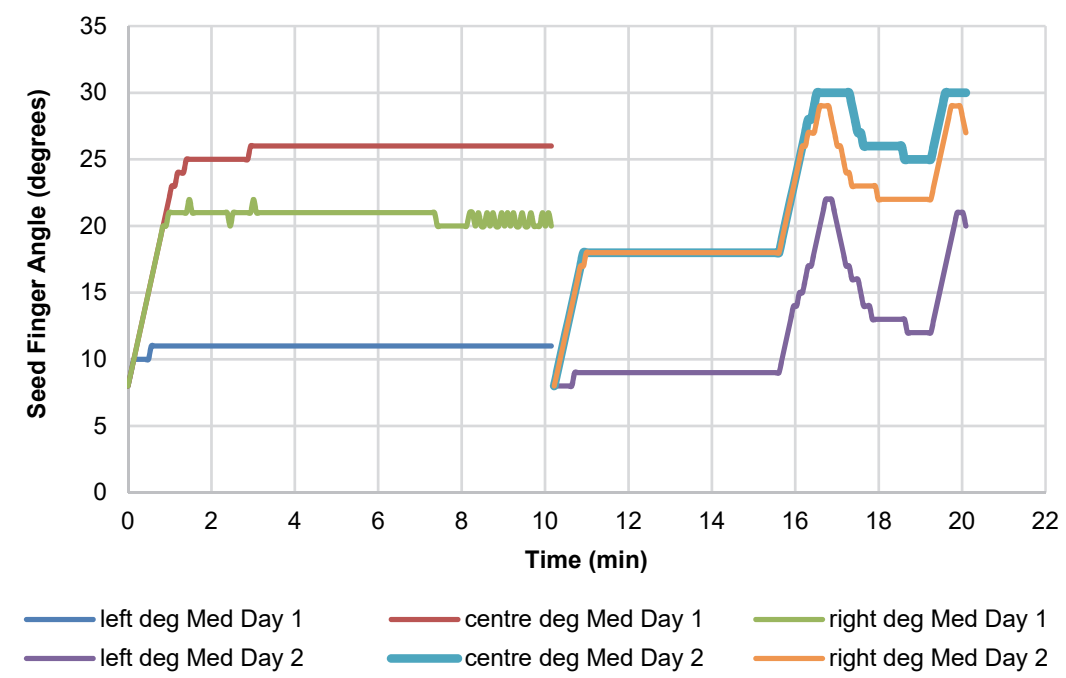

(b)
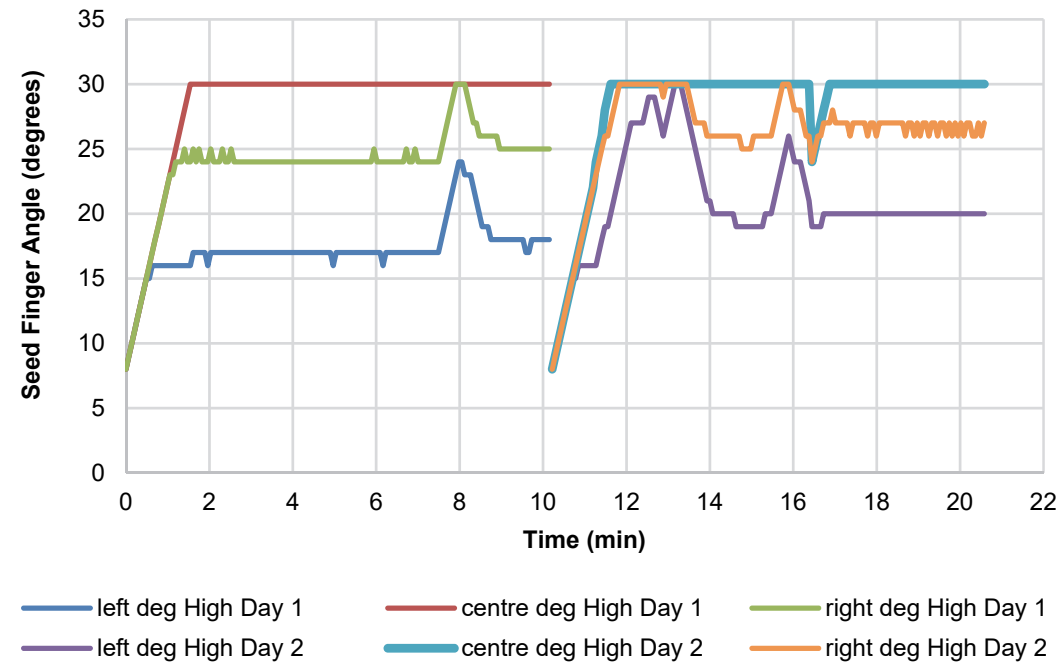

(c)

Figure 3. Seed finger partition movements (degrees) in (a) low, (b) medium, and (c) high auto modes. The three auto modes were run consecutively from low to high for prescribed periods on two trial days. 

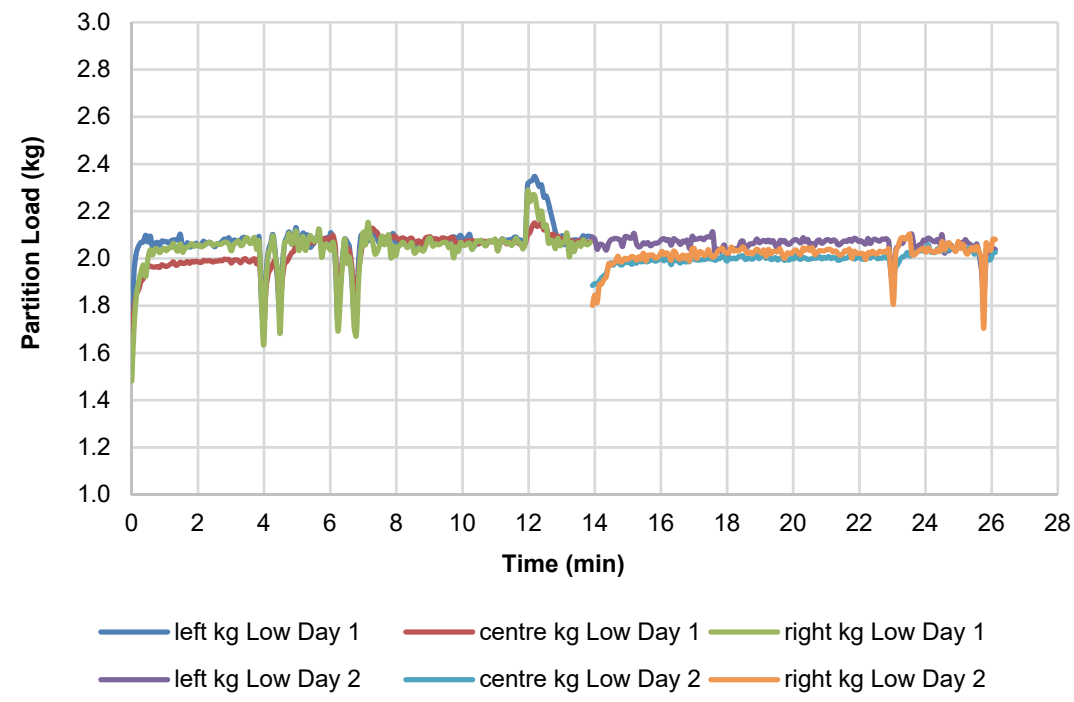

(a)
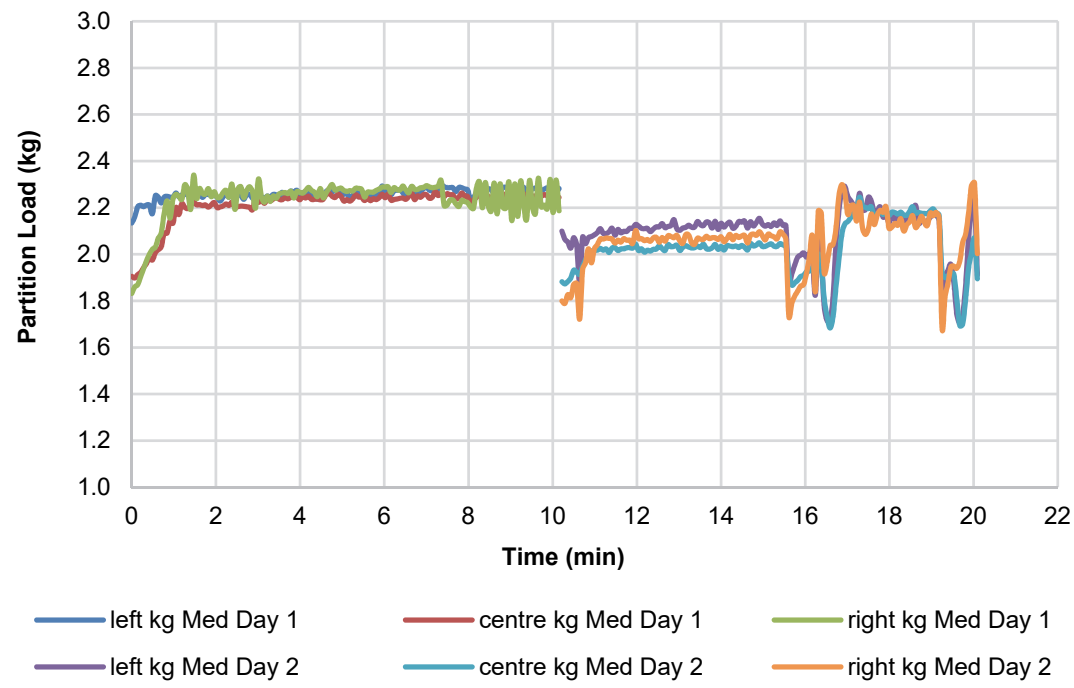

(b)
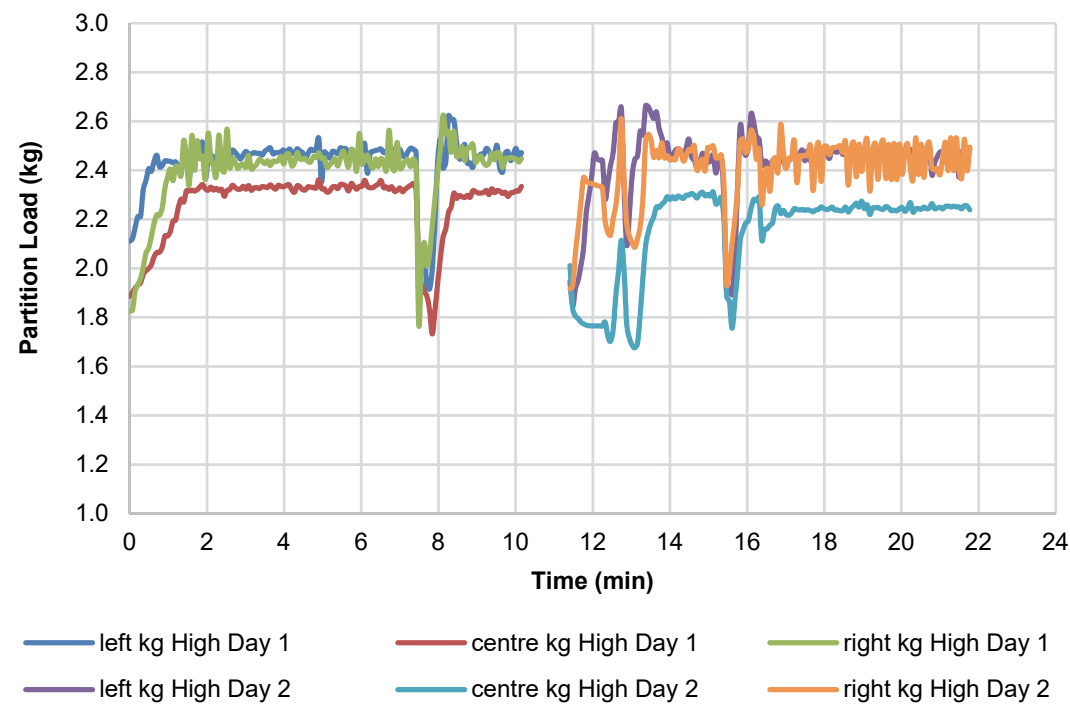

(c)

Figure 4. Seed finger partition loads (kg) during (a) low, (b) medium, and (c) high auto modes. The auto modes were run consecutively from low to high for prescribed periods on two trial days. 
Table 2. Means and standard deviations of angle, load, and current measurements in each auto mode across consecutive trials over two days.

\begin{tabular}{ccccccc}
\hline & $\begin{array}{c}\text { Left Angle } \\
(\text { degrees })\end{array}$ & $\begin{array}{c}\text { Center Angle } \\
(\text { degrees })\end{array}$ & $\begin{array}{c}\text { Right Angle } \\
(\text { degrees })\end{array}$ & $\begin{array}{c}\text { Left Load } \\
(\mathrm{kg})\end{array}$ & $\begin{array}{c}\text { Center Load } \\
(\mathrm{kg})\end{array}$ & $\begin{array}{c}\text { Right Load } \\
(\mathrm{kg})\end{array}$ \\
\hline Mode & $7.8 \pm 2.2$ & $16.5 \pm 2.8$ & $15.4 \pm 1.9$ & $2.07 \pm 0.07$ & $2.02 \pm 0.06$ & $2.03 \pm 0.08$ \\
Low & $11.4 \pm 2.9$ & $23.2 \pm 4.8$ & $20.3 \pm 3.4$ & $2.17 \pm 0.12$ & $2.12 \pm 0.14$ & $2.15 \pm 0.14$ \\
Medium & $19.4 \pm 3.5$ & $29.1 \pm 3.5$ & $25.5 \pm 3.2$ & $2.43 \pm 0.14$ & $2.20 \pm 0.17$ & $2.40 \pm 0.14$ \\
High & & $151.7 \pm 1.2$ & $153.4 \pm 1.7$ \\
\hline
\end{tabular}

Bagshaw (2011) measured higher input and discharge rates at the right and left ends of the gin stand, which were not controlled by individualized partitions, and lower rates across the center of the gin stand, which was nominally denser and formed no or little seed roll burst point from which seed could be discharged. Bagshaw (2011) concluded that uneven loading of seed cotton into the roll box created movement of seed cotton from high-density areas to neighboring lower-density areas, noting that ginned seed discharge (burst point) was facilitated by the end plates of the roll box and seed conveyor tubes within the seed roll.

The proposed effect of the auto mode system is that it should assist in forming and managing an even seed roll density across the gin stand, which in turn should provide benefits in gin turn-out, fiber properties, and reduced energy consumption, as measured by Krajewski et al. (2016). The effect of the auto mode system on seed and fiber properties is discussed later under Residual Lint, Seed Damage, and Fiber Quality Effects.

\section{ENERGY CONSUMPTION}

Gin motor current draw is often used to manage gin production speeds, the setting of seed finger partitions, and ginned seed and lint quality, e.g., residual lint and trash content. Figures $5 \mathrm{a}$ and $5 \mathrm{~b}$ show the relationship between current draw and each auto mode over the two trial days. Figure 5 a shows the current draw over the six consecutive auto mode runs, and figure $5 \mathrm{~b}$ shows the relationship between current draw and measured load, which is a function of the partition angle and seed roll density. Evident in both figures is the high variability in current draw, which increased slightly but not significantly with load setting (fig. 6). The responses observed in these trials differ from earlier observations, where it was noted that as the load applied by the seed fingers was increased in unison, the gin motor current draw at near full production ( 15 bales per hour) decreased linearly at a rate of nearly 12 amps per $\mathrm{kg}$ of force applied. This suggests that the increased load created a seed roll that was more compact and able to rotate cleanly without any impingement of its rotation.

In this study, in which the seed finger partitions responded individually to a nominal load rather than being applied unilaterally, the current draw was not significantly different across the three modes, although there was a small increase in current draw from the low $(151.2 \mathrm{~A})$ to medium $(151.4 \mathrm{~A})$ to high $(153.4 \mathrm{~A})$ modes. The increased current draw in high mode was likely an effect of the increased friction between the seed roll and the partitions, arising from greater unevenness between the partitions across the gin stand, and this effect diminished at lower loads. Motor current draw is specific to an individual gin stand and will vary considerably with feed volume and seed cotton quality (e.g., trash level), ginning conditions (e.g., seed cotton moisture), and gin stand maintenance (e.g., gin saw sharpness).

\section{Residual Lint, SeEd DAMAge, AND FibER QUALITY EFFECTS}

Table 3 lists the ANOVA results for the effects of the three tested factors (trial day, partition position, and auto mode) on the measured responses (residual lint and seed damage), and figure 7 shows the main effects plots for residual lint and seed damage. The plots show the raw data means of measurements at the different levels of each factor, with a dashed reference line indicating the grand mean of the response data. Residual lint values were significantly affected by partition position $(\mathrm{p}=0.000)$ but not by mode, although a pattern was evident for this effect. Seed falling from the center $(10.7 \%)$ and right $(10.53 \%)$ partitions had much less residual lint than seed from the left partition $(11.31 \%)$, which agrees with the observation that the seed roll on the left side was less dense. While the differences were not significant, the residual lint values were lowest for the partitions operating in high mode (10.63\%), i.e., high density, versus the low (11.04\%) and medium modes (10.88\%), which agrees with the observations reported by Krajewski et al. (2016). No interactions were observed between factors.

Samples from the adjacent gin stand without the separate partitions and auto mode system had a mean residual lint value of $10.9 \%$. Krajewski et al. (2016) observed that residual lint values on the same cotton gin were in excess of $10 \%$ when all partitions were aligned at $0^{\circ}$ and were reduced to $8.6 \%$ at $28^{\circ}$, a difference of $1.4 \%$ at a linear reduction rate of $>0.05 \%$ less lint on the seed per degree of increment. The results for the auto mode system indicate a similar effect in which the seed roll, e.g., across the center and right partitions and during the high mode, was denser and provided lower residual lint values. The results also indicate a possible deficiency in the auto mode system, i.e., the partition movement should be metered more slowly. The $3 \mathrm{~s}$ monitoring period for partition load meant that the system compensated too quickly for low-density zones. The partition angle therefore increased too quickly to find the minimum load on the partition before that section of the seed roll had properly equilibrated with seed cotton.

Seed damage values were low, and the overall values between modes were not significantly different. However, the seed damage values were significantly affected by the partition position. Seed damage was least on the left partition $(2.0 \%)$ and greater on the center $(2.2 \%)$ and right partitions $(2.67 \%)$. Seed damage values were also significantly higher on the modified gin than on the adjacent gin stand $(0.6 \%)$. There was no significant difference between the auto modes. The increased seed damage across partitions may be due to differences in the partition angles and unginned or partially ginned seed cotton being damaged by the large angle gaps 


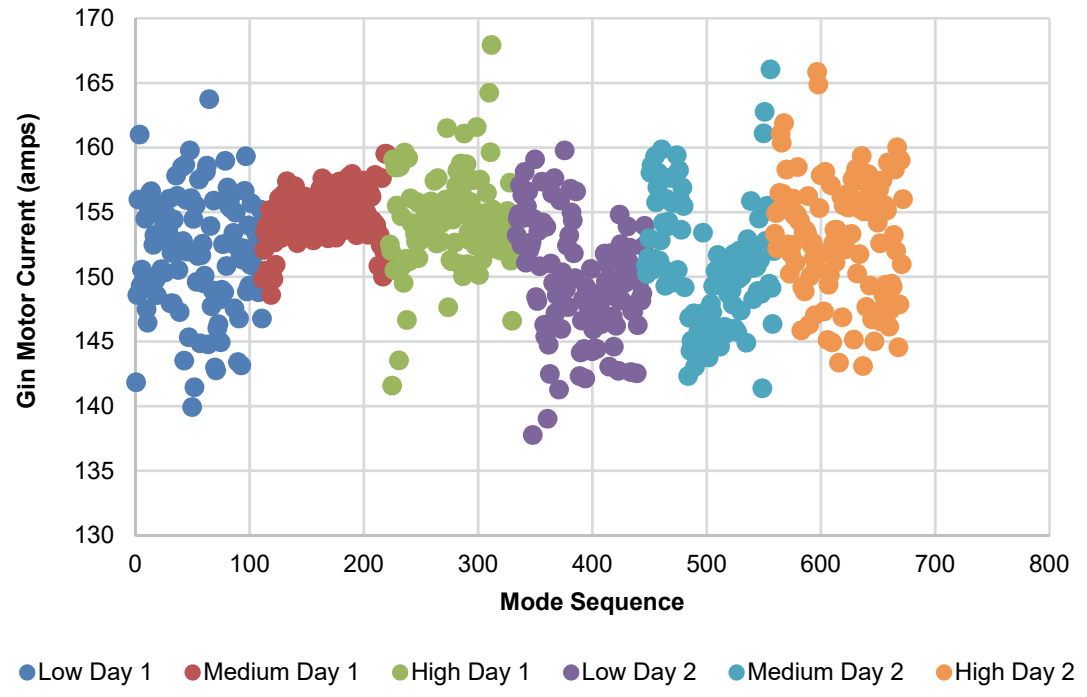

Figure 5a. Gin motor current draw for each auto mode (low, medium, and high). The auto modes were run consecutively for approximately 15 min each day on two trial days.

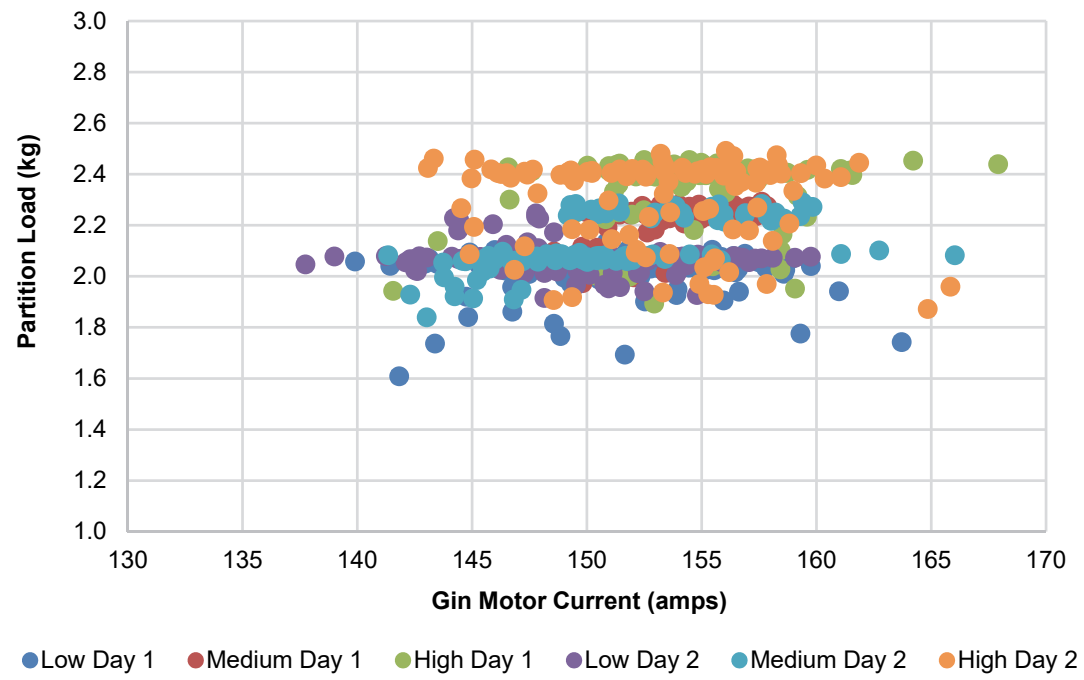

Figure 5b. Gin motor current draw logged for each auto mode (low, medium, and high). The auto modes were run consecutively for approximately 15 min each day on two trial days.

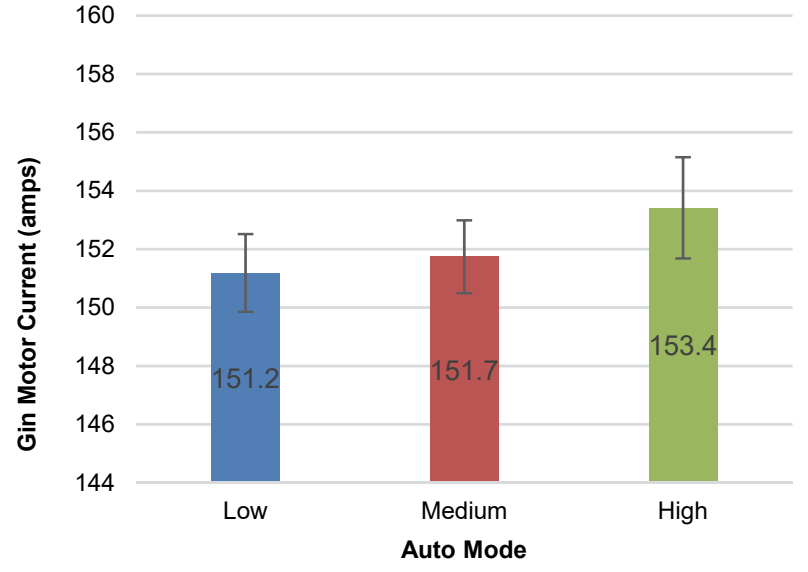

Figure 6. Average current draw from figure 5b for each auto mode (low, medium, and high). Error bars represent one standard deviation of the mean. between partitions. This post-hoc hypothesis reflects the relatively open position of the left partition and the lower density of the seed roll over that partition versus the highly closed positions of the center and right partitions and the subsequently more densely packed seed roll over those partitions.

It is proposed that modulating the extent of partition movement in response to load could improve the seed damage effect and could also reduce the observed slight increase in current draw in high mode. The significant difference in seed damage between trial days does not reflect the applied treatments in the modified gin. The seed damage was very low in these trials compared with values reported by other researchers using the same measurement, e.g., ranging from $9 \%$ to $12 \%$ for different gin feed rates (Moore and Shaw, 1967 ) and from $6 \%$ to $26 \%$ for different moisture levels (Mangialardi et al., 1988; Columbus and Mangialardi, 1996). More trials are required over a wider range of seed 
Table 3. Nested ANOVA results for residual lint and seed damage by trial day (1 or 2), auto mode (low, medium, or high), and partition position (left, center, or right). Significant factors $(\alpha=0.05$ or better) are indicated in bold.

\begin{tabular}{|c|c|c|c|c|c|}
\hline \multirow[b]{2}{*}{ Factor } & \multirow{2}{*}{$\begin{array}{c}\text { Degrees of } \\
\text { Freedom }\end{array}$} & \multicolumn{2}{|c|}{ Residual Lint } & \multicolumn{2}{|c|}{ Seed Damage } \\
\hline & & p-Value & Variance $(\%)$ & p-Value & Variance $(\%)$ \\
\hline Trial day (1 or 2$)$ & 1,53 & 0.547 & 0.00 & 0.011 & 22.34 \\
\hline Auto mode (high, medium, or low) & 4,53 & 0.616 & 0.00 & 0.939 & 0.00 \\
\hline Partition position (left, center, or right) & 12,53 & 0.000 & 65.37 & 0.000 & 43.16 \\
\hline Error $(3 \times$ replicate $)$ & & & 33.63 & & 34.50 \\
\hline
\end{tabular}

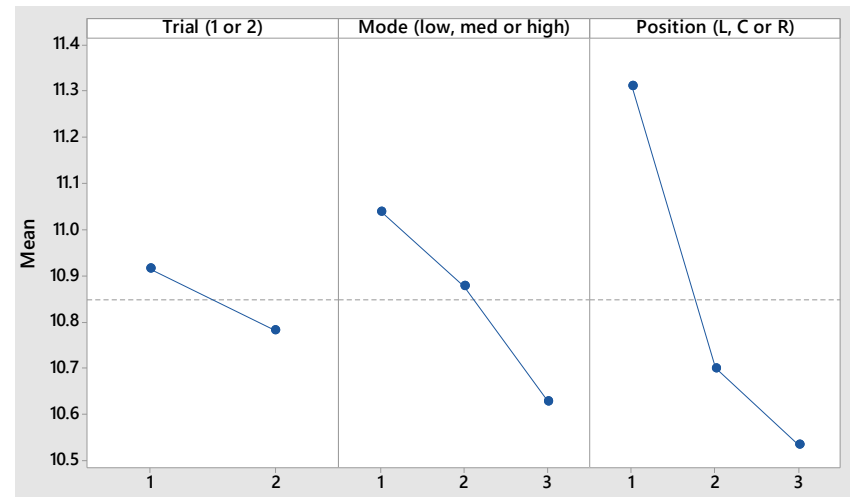

(a)

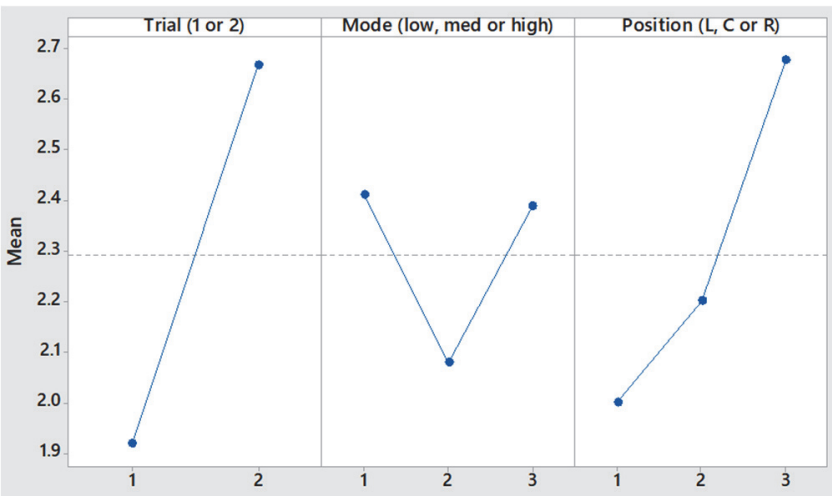

(b)

Figure 7. Percentages of (a) residual lint and (b) seed damage for each trial day (1 or 2$)$, auto mode (1 = low, $2=$ medium, and $3=$ high), and partition (1 = left, 2 = center, and 3 = right $)$.

conditions to properly ascertain the effects on seed damage of non-aligned partition movement across the roll box.

Tables 4 through 7 list the ANOVA results for the effects of the three tested factors on HVI fiber length, strength, color, and trash properties, and figures 8 through 11 show the main effects plots. The low mode had the best and most consistent effect on fiber properties, although the differences were not statistically significant. The low mode achieved the longest (by $0.3 \mathrm{~mm}$ from the control), strongest (by $0.3 \mathrm{~g}$ per tex), whitest (by $0.7 \mathrm{Rd}$ ), and cleanest (by $0.08 \%$ trash area) fiber compared to the other modes and the control. More testing is required to confirm these but they suggest that the seed roll was consistent in density, as enabled by the separately controlled partitions, and of a density that allowed the seed to be properly and evenly raked by the saw teeth while allowing a consistent seed discharge (burst point) from the seed roll. The tightness or density of the seed roll also affected the amount of trash held in the lint after ginning.

\section{CONCLUSIONS}

The research presented in this article provides evidence the position of the seed finger partition influences the amount of residual lint on ginned seed and supports the idea

Table 4. Nested ANOVA results for HVI upper half mean length and short fiber content by trial day (1 or 2), auto mode (low, medium, or high), and partition position (left or right). Significant factors $(\alpha=0.05$ or better) are indicated in bold.

\begin{tabular}{|c|c|c|c|c|c|}
\hline \multirow[b]{2}{*}{ Factor } & \multirow{2}{*}{$\begin{array}{l}\text { Degrees of } \\
\text { Freedom }\end{array}$} & \multicolumn{2}{|c|}{ Upper Half Mean Length } & \multicolumn{2}{|c|}{ Short Fiber Content } \\
\hline & & p-Value & Variance $(\%)$ & p-Value & Variance $(\%)$ \\
\hline Trial day (1 or 2 ) & 1,35 & 0.972 & 0.00 & 0.935 & 0.00 \\
\hline Auto mode (high, medium, or low) & 4,35 & 0.470 & 0.34 & 0.007 & 40.13 \\
\hline Partition position (left or right) & 6,35 & 0.096 & 26.05 & 0.860 & 0.00 \\
\hline Error $(3 \times$ replicate $)$ & 24,35 & & 73.61 & & 59.87 \\
\hline
\end{tabular}

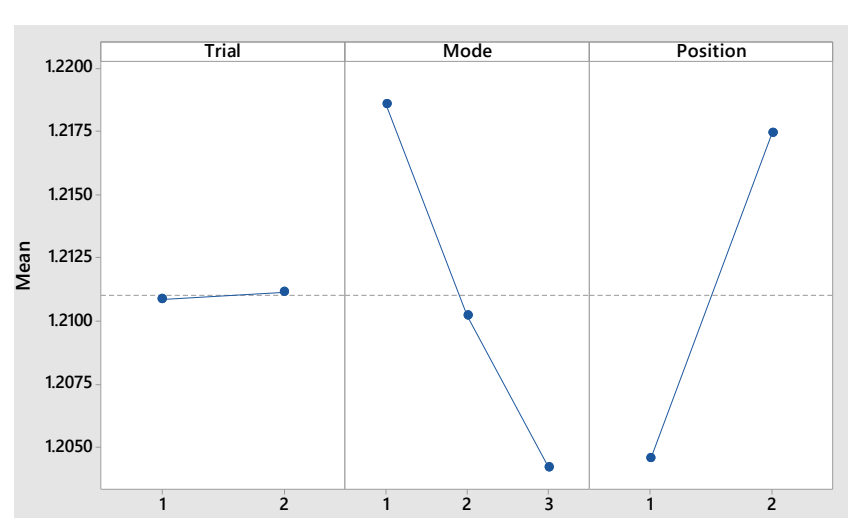

(a)

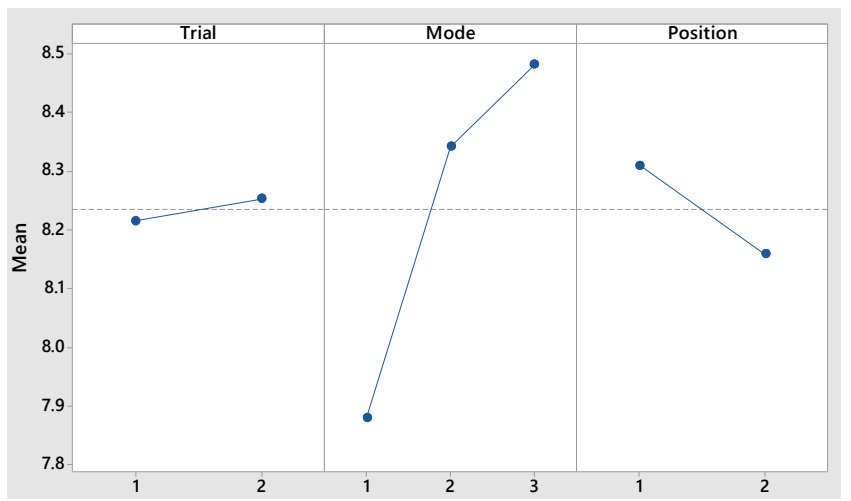

(b)

Figure 8. HVI (a) upper half mean length (inches) and (b) short fiber content (\%) for each trial day (1 or 2$)$, auto mode $(1=$ low, $2=$ medium, and $3=$ high $)$, and partition position $(1=$ left and $2=$ right $)$. 
Table 5. Nested ANOVA results for HVI tenacity and elongation by trial day (1 or 2), auto mode (low, medium, or high), and partition position (left or right). Significant factors $(\alpha=0.05$ or better) are indicated in bold.

\begin{tabular}{|c|c|c|c|c|c|}
\hline \multirow[b]{2}{*}{ Factor } & \multirow{2}{*}{$\begin{array}{l}\text { Degrees of } \\
\text { Freedom }\end{array}$} & \multicolumn{2}{|c|}{ Tenacity } & \multicolumn{2}{|c|}{ Elongation } \\
\hline & & p-Value & Variance $(\%)$ & p-Value & Variance $(\%)$ \\
\hline Trial day (1 or 2 ) & 1,35 & 0.667 & 0.00 & 0.704 & 0.00 \\
\hline Auto mode (high, medium, or low) & 4,35 & 0.313 & 10.44 & 0.210 & 13.57 \\
\hline Partition position (left or right) & 6,35 & 0.150 & 18.15 & 0.498 & 0.00 \\
\hline Error $(3 \times$ replicate $)$ & 24,35 & & 71.41 & & 86.43 \\
\hline
\end{tabular}

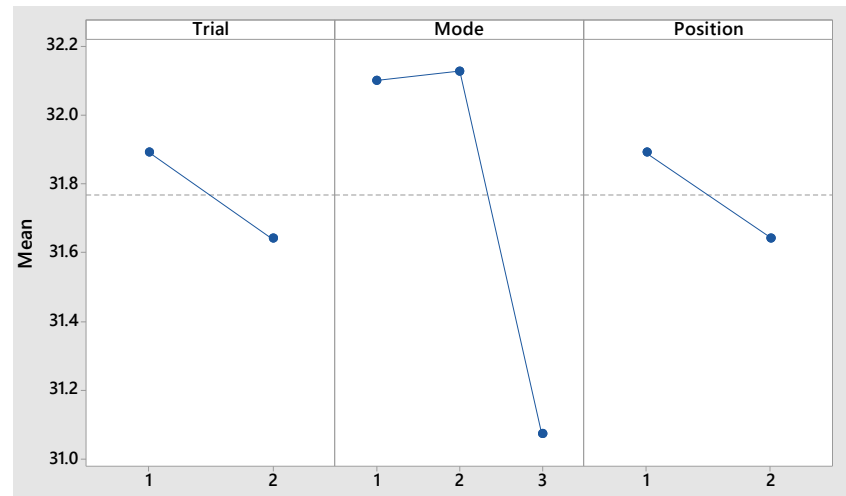

(a)

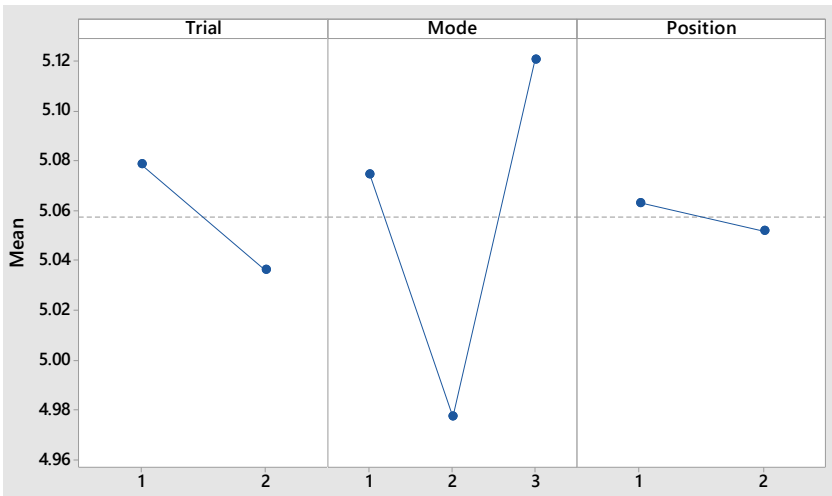

(b)

Figure 9. HVI (a) tenacity (cN per tex) and (b) elongation (\%) for each trial day (1 or 2$)$, auto mode $(1=$ low, $2=$ medium, and $3=$ high), and partition position $(1=$ left and $2=$ right $)$.

Table 6. Nested ANOVA results for HVI reflectance and CIELab yellowness (+b) by trial day (1 or 2$)$, auto mode (low, medium, or high), and partition position (left or right). Significant factors $(\alpha=0.05$ or better) are indicated in bold.

\begin{tabular}{|c|c|c|c|c|c|}
\hline \multirow[b]{2}{*}{ Factor } & \multirow{2}{*}{$\begin{array}{c}\text { Degrees of } \\
\text { Freedom }\end{array}$} & \multicolumn{2}{|c|}{ Reflectance } & \multicolumn{2}{|c|}{ Yellowness } \\
\hline & & $\mathrm{p}$-Value & Variance $(\%)$ & p-Value & Variance $(\%)$ \\
\hline Trial day (1 or 2$)$ & 1,35 & 0.930 & 0.00 & 0.029 & 30.23 \\
\hline Auto mode (high, medium, or low) & 4,35 & 0.266 & 15.34 & 0.778 & 0.00 \\
\hline Partition position (left or right) & 6,35 & 0.090 & 22.81 & 0.029 & 26.86 \\
\hline Error $(3 \times$ replicate $)$ & 24,35 & & 61.85 & & 42.91 \\
\hline
\end{tabular}

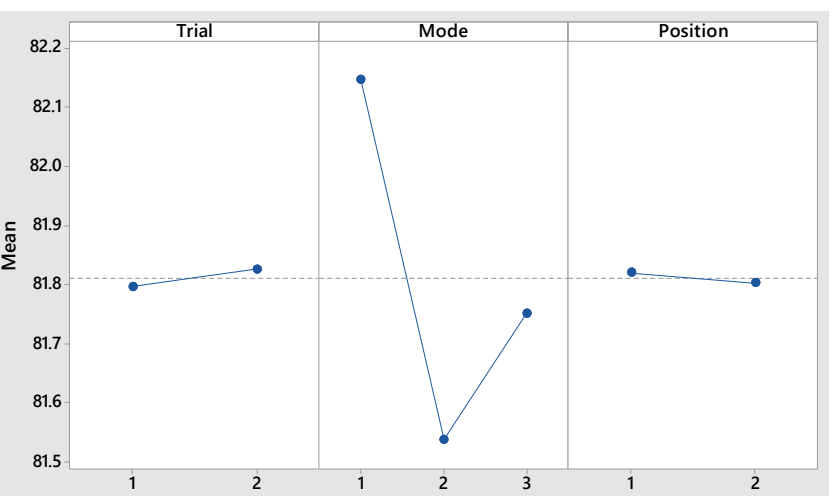

(a)

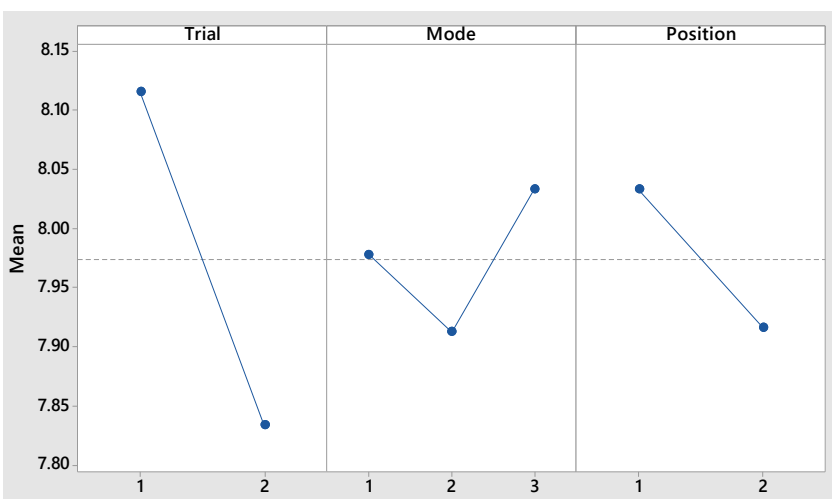

(b)

Figure 10. HVI (a) reflectance and (b) CIELab yellowness $(+b)$ for each trial day $(1$ or 2$)$, auto mode $(1=l o w, 2=$ medium, and $3=$ high $)$, and partition position $(1=$ left and $2=$ right $)$.

that self-adjusting seed fingers could be used to extract more fiber (increased gin turn-out) and improve fiber quality (e.g., trash and fiber length parameters). The dynamic responses of the seed finger partitions to changes in the force exerted on them by the seed roll were measured and examined for their effect on energy consumption (motor current draw), residual lint, seed damage, and fiber properties. The measurements showed that the seed roll density is highly dynamic and dependent on the feed rate and uniformity of the seed cotton distribution in the gin stand.
A load bias to the left side of the gin stand was shown. The left partition was consistently lower than the center and right partitions in all auto modes. Individual partition movements relative to the other partitions were less extreme in the low mode. The low mode also produced the best fiber quality in terms of trash and fiber length and had the lowest energy consumption, although the results were not significantly different from the other two modes or the control. All auto modes produced higher levels of seed damage. This was attributed to extreme deviations in partition alignment across the gin stand, which in turn created sticking points for 
Table 7. Nested ANOVA results for HVI trash count and trash area by trial day (1 or 2), auto mode (low, medium, or high), and partition position (left or right). Significant factors $(\alpha=0.05$ or better) are indicated in bold.

\begin{tabular}{|c|c|c|c|c|c|}
\hline \multirow[b]{2}{*}{ Factor } & \multirow{2}{*}{$\begin{array}{l}\text { Degrees of } \\
\text { Freedom }\end{array}$} & \multicolumn{2}{|c|}{ Trash Count } & \multicolumn{2}{|c|}{ Trash Area } \\
\hline & & p-Value & Variance $(\%)$ & p-Value & Variance $(\%)$ \\
\hline Trial day (1 or 2$)$ & 1,35 & 1.000 & 0.00 & 0.771 & 0.00 \\
\hline Auto mode (high, medium, or low) & 4,35 & 0.052 & 26.92 & 0.099 & 15.05 \\
\hline Partition position (left or right) & 6,35 & 0.695 & 0.00 & 0.815 & 0.00 \\
\hline Error $(3 \times$ replicate $)$ & 24,35 & & 73.08 & & 84.95 \\
\hline
\end{tabular}

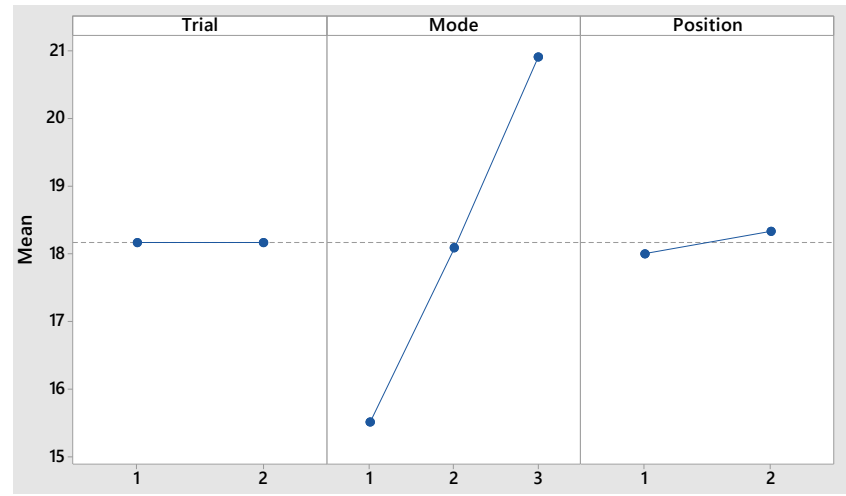

(a)

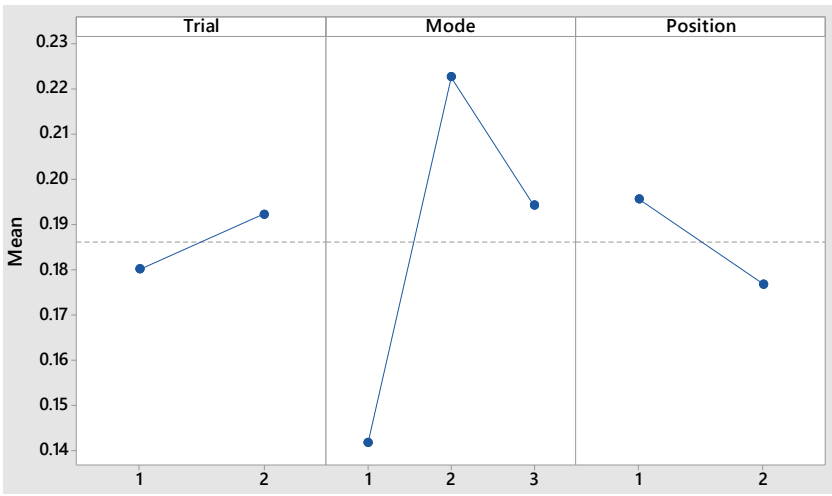

(b)

Figure 11. HVI (a) trash count (number) and (b) trash area (\%) for each trial day (1 or 2$)$, auto mode $(1=$ low, $2=$ medium, and $3=$ high $)$, and partition position $(1=$ left and $2=$ right $)$.

partially ginned seed against the rotating seed roll. More work is required to moderate the auto mode response to load changes across the partitions. However, the trials reported here show that a moderated auto mode system that reflects the partition load, energy consumption, and seed cotton flow may have benefits in fiber quality, residual lint (gin turnout), and/or energy consumption.

\section{ACKNOWLEDGEMENTS}

We gratefully acknowledge financial support from the Australian Cotton Research and Development Corporation for project CMSE1402 and CSIRO Manufacturing. We would also like to thank Auscott Limited and in particular Wayne Towns, David Pratt, and their team for hosting and helping with the trials described in this article. We also gratefully acknowledge Neale Gibbons for his workmanship on the modified system, Michel Givord and Ian Redknap (deceased) from CSIRO Manufacturing for their design work.

\section{REFERENCES}

Bagshaw, K. (2011). Improving gin stand performance to benefit Australian cotton. MS thesis. Toowoomba, QLD, Australia: University of Southern Queensland, School of Agricultural Engineering.

Columbus, E. P., \& Mangialardi Jr., G. J. (1996). Cottonseed moisture and seed damage at gins. Trans. ASAE, 39(5), 16171621. https://doi.org/10.13031/2013.27677

Krajewski, A., Gordon, S. G., \& Fox, D. (2016). A self-adjusting seed finger system to improve gin turnout. Trans. ASABE, 59(6), 1863-1871. https://doi.org/10.13031/trans.59.11404

Mangialardi Jr., G. J., Bargeron III, J. D., \& Rayburn Jr., J. S. (1988). Gin-stand feed rate effects on cotton quality. Trans. ASAE, 31(6), 1844-1850. https://doi.org/10.13031/2013.30945

Moore, V. P., \& Shaw, C. S. (1967). Mechanical damage to cottonseed: Ginning effects. Cotton Gin Oil Mill Press, 68(5), $10-11$. 